\title{
Development and Preclinical Study of Free Radical Imaging Using Field-Cycling Dynamic Nuclear Polarization MRI
}

\author{
Hideo Utsumi ${ }^{1,2}$, Toshiki Masumizu ${ }^{1,2}$, Ryoma Kobayashi ${ }^{1,3}$, Tomoko Tahira ${ }^{1,4}$, \\ Fuminori Hyodo ${ }^{1.5}$, Tatsuya Shimizu ${ }^{6}$, Tatsuya Naganuma ${ }^{7}$, Kazunori Anzai ${ }^{8}$ \\ ${ }^{1}$ Innovation Center for Medical Redox Navigation, Kyushu University, Fukuoka 812-8582, Japan \\ ${ }^{2}$ School of Pharmaceutical Sciences, University of Shizuoka 422-8526, Japan \\ ${ }^{6}$ Department of Radiology, School of Medicine, University of Yamanashi, Yamanashi 409-3898 Japan \\ ${ }^{7}$ Japan Redox Limited, Fukuoka 812-0044, Japan \\ ${ }^{8}$ Faculty of Pharmaceutical Sciences, Nihon Pharmaceutical University, Saitama 362-0806, Japan \\ *E-mail; utsumih@u-shizuoka-ken.ac.jp
}

Contents:

Supplementary Figure S1

Supplementary Figure S2

Supplementary Figure S3

Supplementary Figure S4

Supplementary Figure S5 
(A)
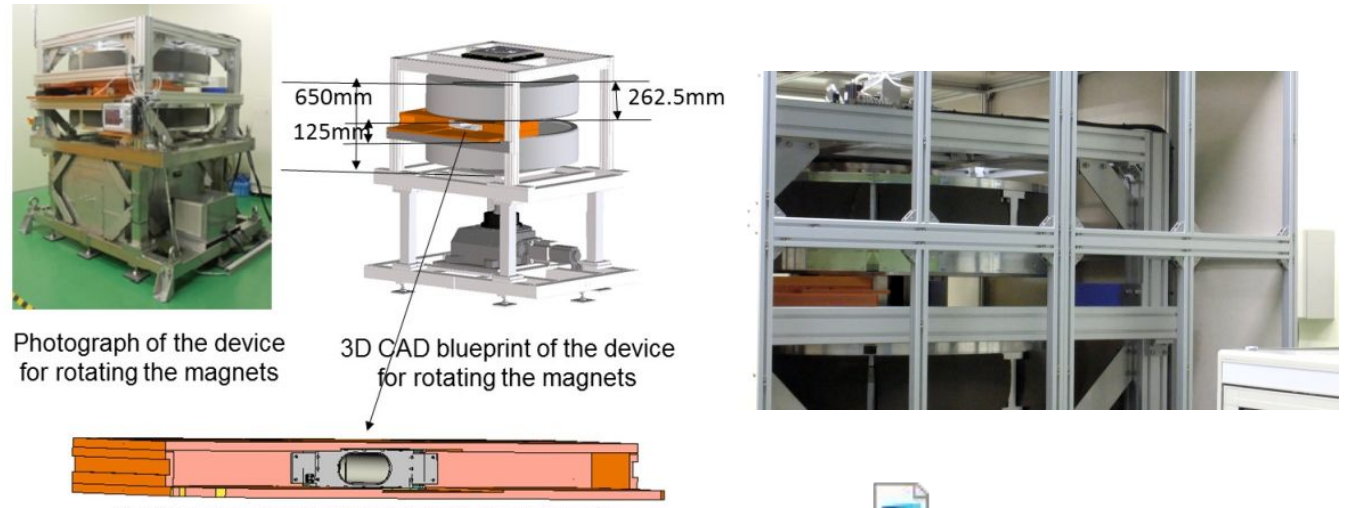

Holding plate for NMR coil \& field gradient coils

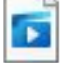

C 6-2.MOV

(B)

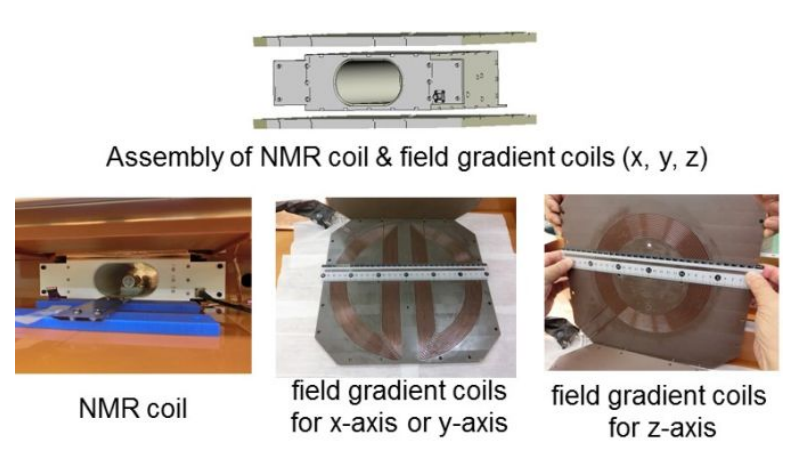

\section{Supplementary Figure S1}

(A) The picture and 3D CAD blueprint of the device for rotating the magnets and movie.

(B) 3D CAD blueprint and the picture of the NMR coil and the field gradient coils for $\mathrm{x}-$, y-, and z-axis. 
(A)

(B)

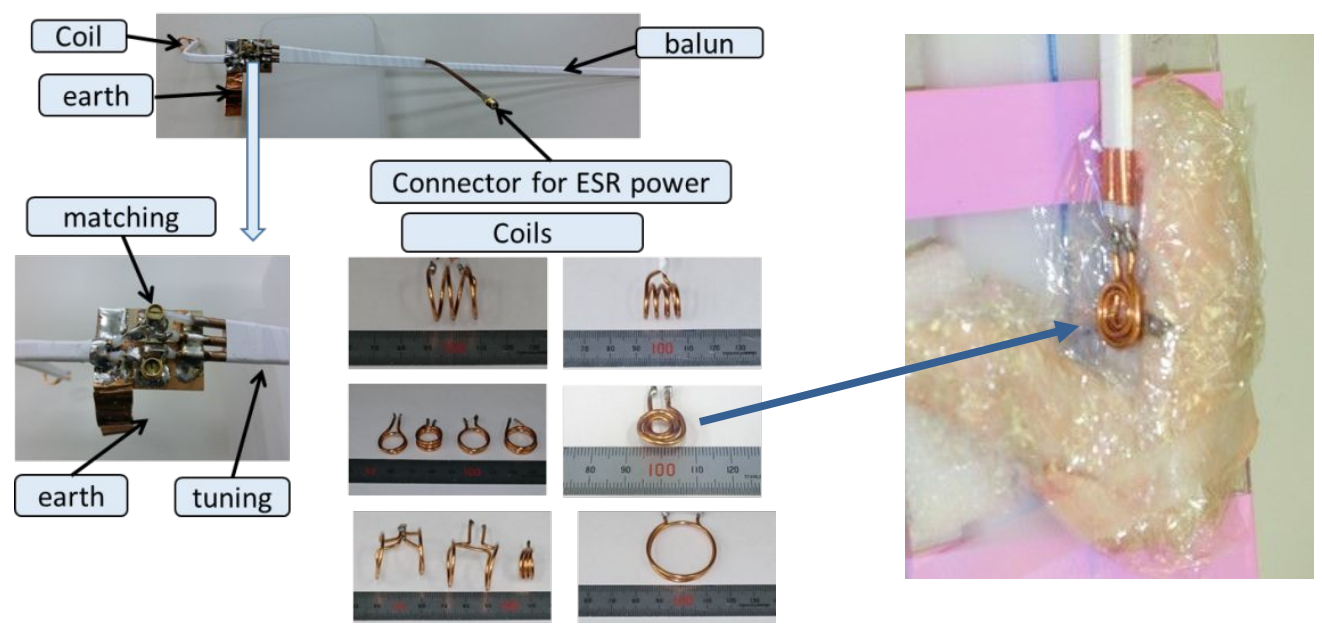

\section{Supplementary Figure S2}

(A) The various types of surface coils were prepared for ESR irradiation, according to the previous report (Matsumoto, S.; Nagai, M.; Yamada, K.; Hyodo, F.; Yasukawa, K.; Muraoka, M.; Hirata, H.; Ono, M.; Utsumi, H. A Composite Resonator Assembly Suitable for EPR/NMR Coregistration Imaging. Concepts Magn. Reson. Part B: Magn. Reson. Eng. 2005, 25B (1), 1-11.). The ESR frequency of all the coils was adjusted to approximately $136-137 \mathrm{MHz}$.

(B) The spiral coil was attached on the skin where the small cup (Hegenberg 0516760, 8 $\mathrm{mm}$ height and outer diameter), containing $2 \mathrm{mM} \mathrm{Cmp}$, was embedded into the raw chicken wing. We then performed FC-DNP-MRI at various ESR irradiation powers. 


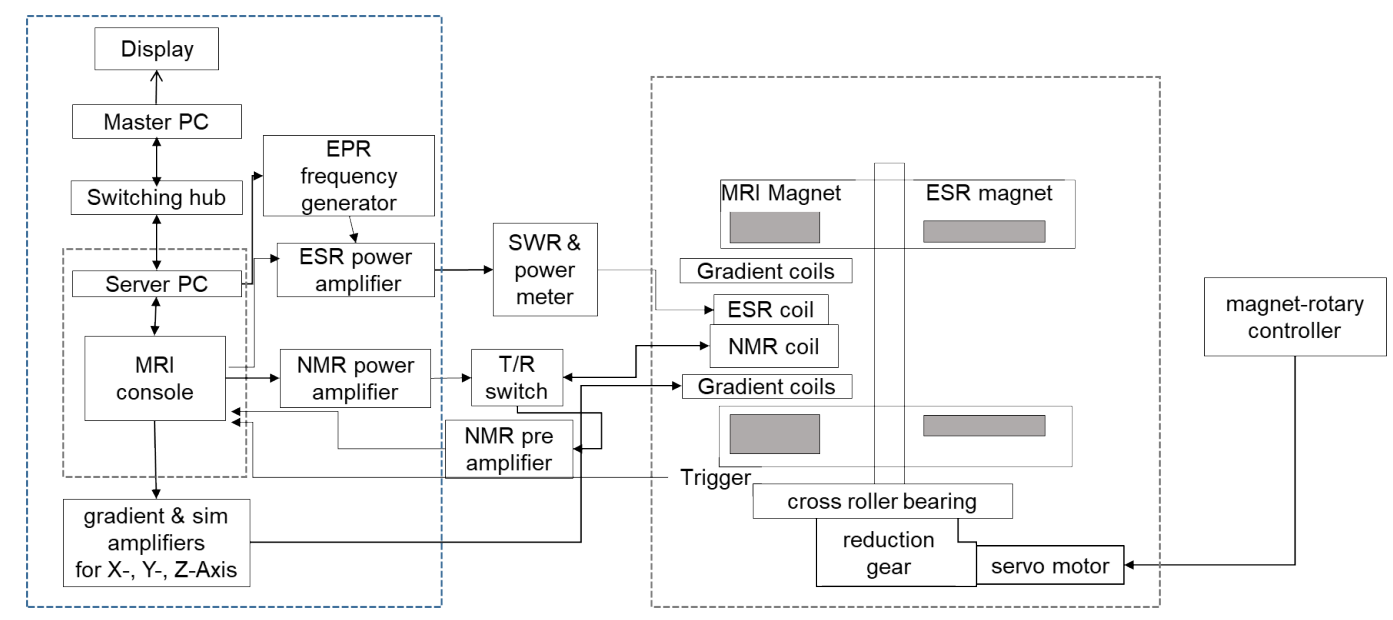

\section{Supplementary Figure S3}

Block diagram of the novel FC-DNP-MRI. 


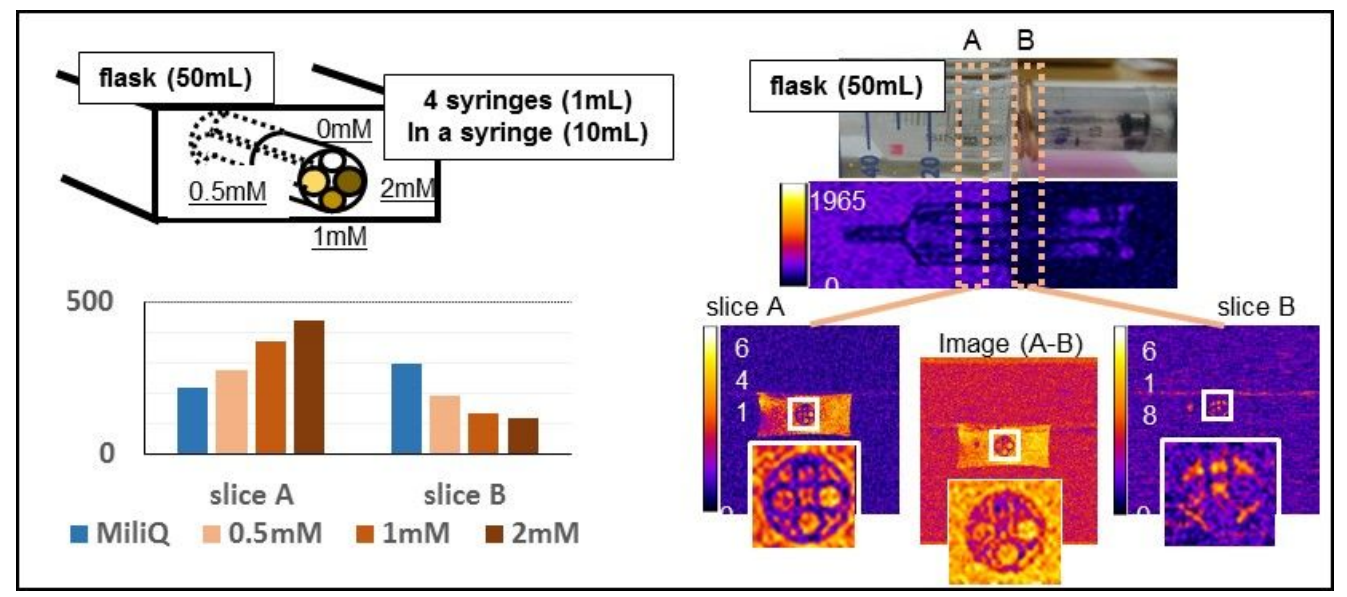

\section{Supplementary Figure S4}

DNP-MRI images of different concentrations of Cmp with the novel FC-DNP-MRI system and one-turn ESR coil. (A) The phantom consisted with the flask (50 mL), syringe $(10 \mathrm{~mL})$ and 4 tuberculin syringes $(5.5 \mathrm{~mm}$ of inner diameter) containing pure water, 0.5 $\mathrm{mM}, 1 \mathrm{mM}$, and $2 \mathrm{mM} \mathrm{Cmp}$, respectively, and MR images obtained in the coronal (upper) and transverse direction (lower) with a 3-slice sequence $(5 \mathrm{~mm}$ thickness and $1 \mathrm{~mm}$ interval).

The influence of free radical concentration on the image contrast intensity was clearly demonstrated by comparing the image between the slice $\mathrm{A}$ and $\mathrm{B}$, with and without the ESR coil, respectively. The signal intensities of the ROI at the 4 tubes filled with $0,0.5$, 1.0 , and $2.0 \mathrm{mM}$ of $\mathrm{Cmp}$ solution in the slice A and B were obtained and plotted at the bottom left. The ESR irradiation decreased the signal intensity in slice A as compared to that in slice B in a dose-dependent manner. 

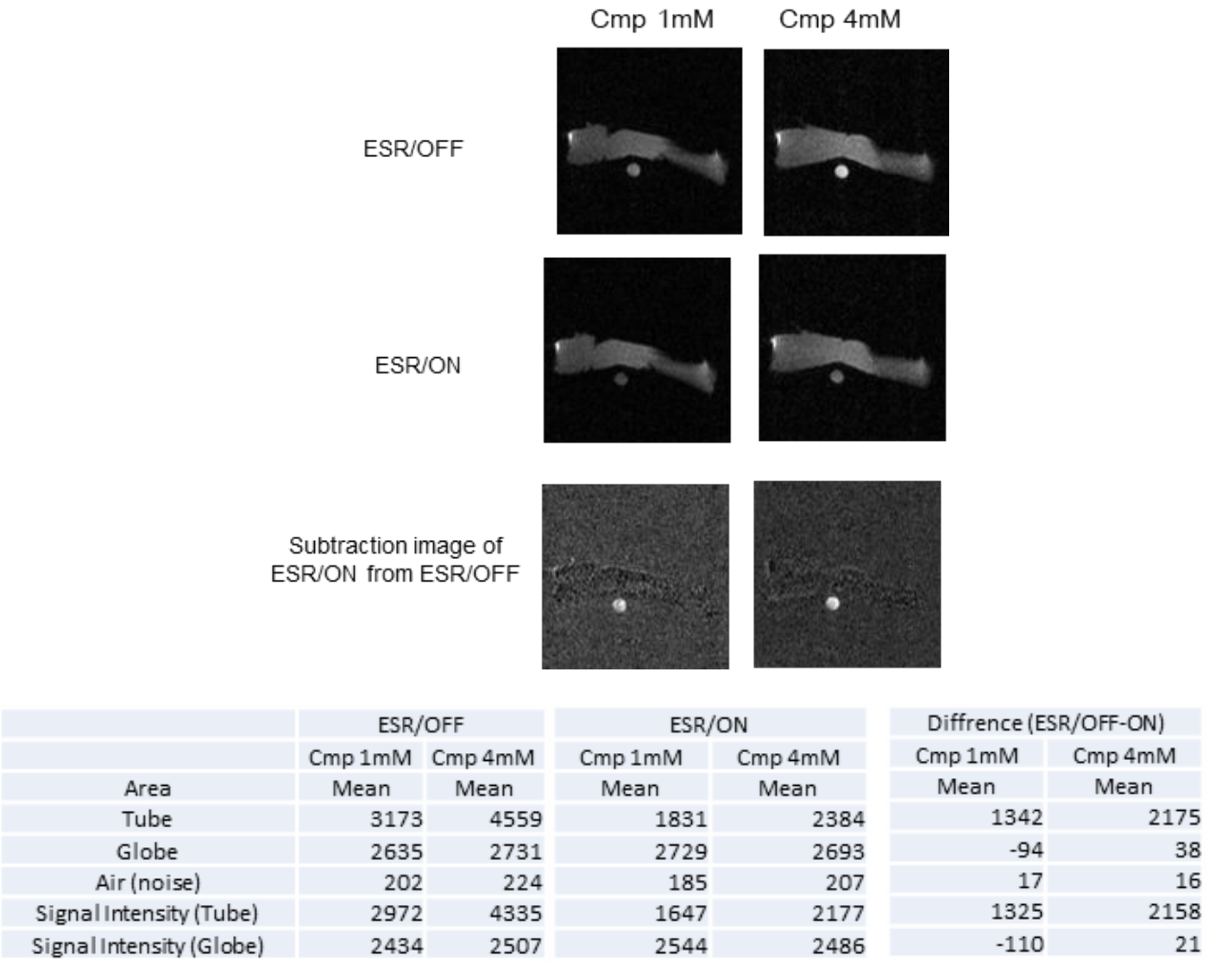

\section{Supplementary Figure S5}

We prepared the phantom consisting of two Agilent tubes $(410090,8 \mathrm{~mm}$ of inner diameter, $30 \mathrm{~mm}$ in length) containing either 1 or $4 \mathrm{mM} \mathrm{Cmp}$, located under a kitchen hand rubber glove filled with poly-vinyl alcohol, and examined the pulse sequence and heat stress caused during FC-DNP-MRI. By optimizing the FC-DNP-MRI conditions, a clear free radical image and signal intensity was obtained, and the signal intensities are indicated in the above Table. The heat stress was observed to be less than $2{ }^{\circ} \mathrm{C}$ of the temperature under the glove. 